\title{
Variation in the Origins of the Branches of the Axillary Artery
}

\author{
DONALD F. HUELKE \\ Department of Anatomy, The University of Michigan Medical School, \\ Ann Arbor, Michigan
}

Variability in the origin of certain branches of the axillary artery is frequently apparent when demonstrating the system in the dissecting room. The variations of these branches have not been entirely explained in previous studies, nor have the findings of these investigations been uniform. It is the purpose of this study to present the variations in origin of the branches of the axillary artery with a view to clarification of both the "regularity" and "variability" in these vessels.

\section{MATERIAL}

The data for this report were recorded from 89 adult cadavers: 79 Whites (60 males and 19 females) and 10 Negroes ( 7 males and 3 females). Both the right and left sides were used so that a total of 178 sides were observed. This material was also used in a previous report on certain branches of the subclavian artery (Huelke, '58).

\section{OBSERVATIONS AND RESULTS}

The typical axillary artery is said to give origin to 6 branches: superior thoracic, thoraco-acromial, lateral thoracic, subscapular, and anterior and posterior circumflex humeral arteries. Other unnamed branches are also present.

The superior thoracic artery (fig. 1) was present in $97.8 \%$ of all sides, usually as a branch of the first part of the axillary artery ( $86.6 \%$, table 1). Rarely the superior thoracic artery originated from the terminal part of the subclavian artery, from the second part of the axillary artery, or from the thoraco-acromial or lateral thoracic arteries. In table 1 some of the previous investigations on the superior thoracic artery are also presented. ${ }^{1}$ DeGaris and Swartley ('28) reported that the superior thoracic artery arose from the first part of the axillary artery in $96.9 \%$ of cases. In their study it arose from the thoracoacromial artery in $3.1 \%$. P'an ('40), and Trotter et al. ('30) found that the superior thoracic artery arose directly from the axillary artery in almost all cases. Pellegrini ('06) found that this artery had a direct axillary origin in 9 out of 10 sides. The specific part of the axillary artery from which the superior thoracic artery arose was not stated in these papers. Adachi ('28), without mentioning the exact site of origin, reported that the superior thoracic artery came directly from the axillary artery in $70 \%$; in $30 \%$ it originated from other arteries, most frequently from the thoraco-acromial artery.

The thoraco-acromial artery was a branch of the second part of the axillary artery in two-thirds of the sides in my series (fig. 1A) and arose from the axillary artery medial to the pectoralis minor tendon in approximately the other one-third (table 2, fig. 1B). The thoracoacromial artery usually arises near the medial border of the pectoralis minor muscle rather than directly behind or towards its lateral border. Other sites of origin are rare. On one side the thoracoacromial artery arose from the brachial artery. In this instance the second part of the axillary artery bifurcated into the brachial and deep brachial arteries with the thoraco-acromial artery arising from the brachial artery adjacent to the lateral edge of the pectoralis minor muscle. No

${ }^{1}$ Throughout this study reference is made only to those articles in which a significant number of axillary arteries ( 100 or more sides) were used. Textbooks and articles not mentioning the number of cases studied or which relied on terms such as frequently, usually, rarely, etc., in place of actual percentage figures, are not included. 
TABLE 1

The sites of origin of the superior thoracic artery

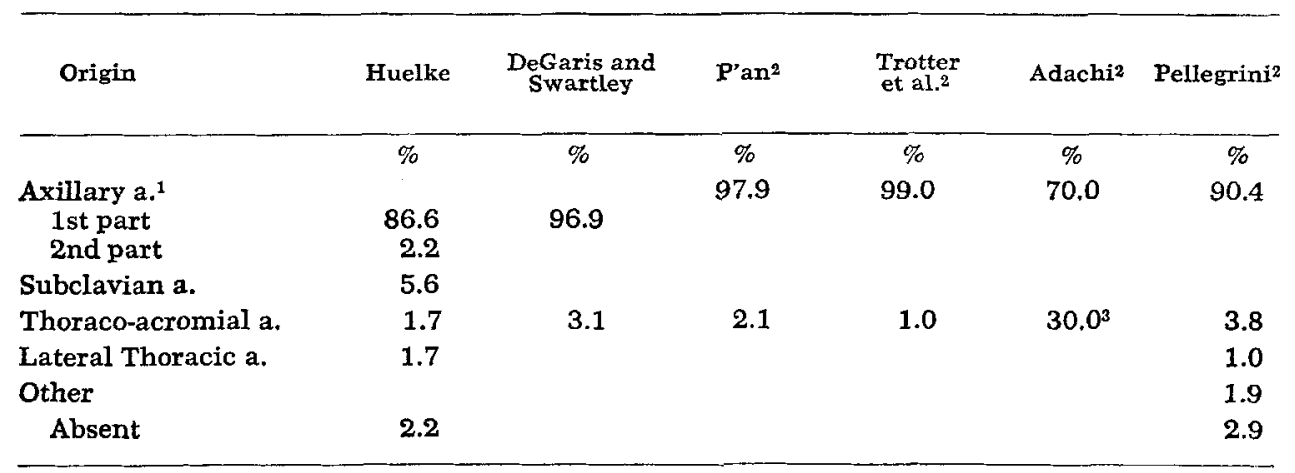

I The axillary artery was considered to be divided into three parts by the tendon of the pectoralis minor muscle. The first part of the artery lies medial to the muscle; the second part is behind it, and the third part is lateral to it and extends to the lower border of the teres major muscle.

2 These authors did not indicate the specific part of the axillary artery from which the branch originated; however, in some instances in P'an's study the sites of origin of the vacious branches were estimated from his illustrations.

3 "It (the superior thoracic artery) frequently originates from other arteries, most frequently from the thoraco-acromial artery"-Adachi.

true thoraco-acromial artery was found on two sides $(1.1 \%)$. In these cases the artery was split-one part arose from the axillary artery medial to the pectoralis minor tendon and the other part from the axillary artery behind this muscle. In addition to its usual branches, the thoracoacromial artery infrequently gives rise to the superior thoracic and lateral thoracic arteries (tables 1 and 3 ).

These findings on the origin of the thoraco-acromial artery do not agree with the results of DeGaris and Swartley (table 2). DeGaris and Swartley found that the thoraco-acromial artery arose most frequently from the first part of the axillary artery and from the second part of the axillary artery in only $12.1 \%$ of cases. No comparisons of data can be made with the other investigators, for the part of the axillary artery which was the most frequent site of origin was not given in the studies of P'an, Trotter et al., Adachi or Pellegrini. All authors agree that the thoraco-acromial artery is almost invariably a direct branch of the axillary artery and that its origin is usually separate from that of any other axillary branch.

The lateral thoracic artery (table 3) had a direct origin from the axillary artery in two-thirds of the sides examined, arising from the second part of the axillary artery more often (52.2\%, fig. 1A) than from the first $(10.7 \%)$ or third part $(1.7 \%)$. Rarely $(6.7 \%)$ the lateral thoracic artery arose from the thoraco-acromial artery. When the lateral thoracic artery did not arise directly from the axillary artery or from the thoraco-acromial artery it was a branch of the subscapular artery or of one of its terminals - the thoracodorsal artery (fig. 1B). This type of origin was seen in $28.7 \%$ of sides. Such a lateral thoracic artery may be a discrete and definite branch or it may appear to be an enlargement of some of the thoracic branches of the thoracodorsal artery. These thoracic branches are always present; they are enlarged when no specific lateral thoracic artery from a more typical origin is present.

Briefly then, the lateral thoracic artery arose most frequently from the second part of the axillary artery and was more often a direct branch of the axillary artery than a branch of another artery. Such is also the case when it had an origin from the first part of the axillary artery. However, 


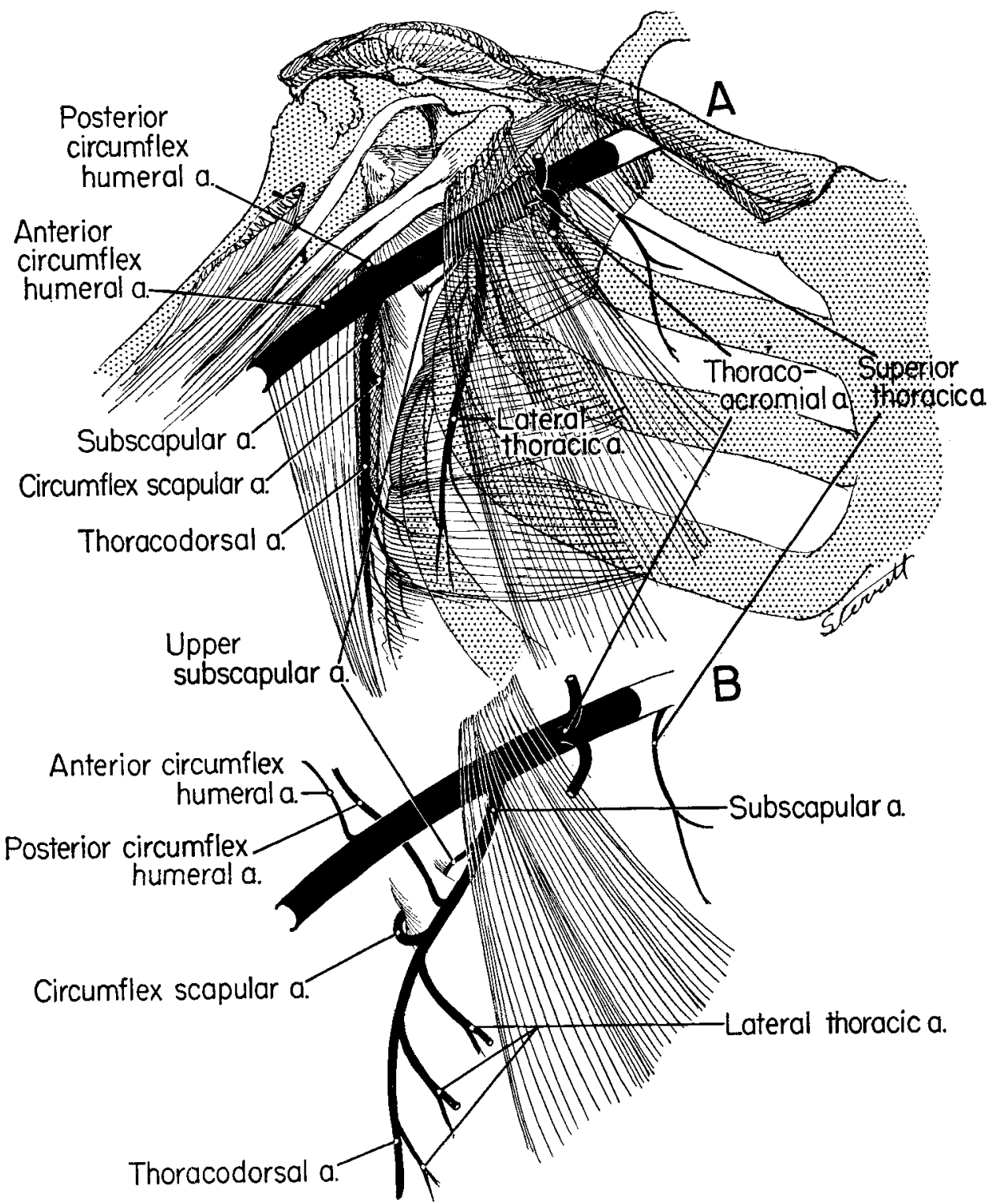

Fig. 1 Composite illustrations of the axillary branches showing: (A) the most frequent site of origin of each branch, and (B) the second most frequent site or origin of each of the axillary branches.

when arising at the level of the third part of the axillary artery, the lateral thoracic artery was more often a branch of the subscapular or thoracodorsal artery than a direct axillary branch. ${ }^{2}$

2 Because the structures of the anterior axillary wall had already been dissected in my specimens the Iateral mammary arteries could not be identified with certainty, and thus actual percentages were not calculated. However, branches of the
In certain respects the data of DeGaris and Swartley, on the lateral thoracic artery, are quite similar to those of this study (table 3). Their data also show that when the lateral thoracic artery arises from

lateral thoracic artery were found to pass into the subcutaneous tissue near the lateral edge of the pectoralis major muscle, and it is assumed that at least some of these were lateral mammary arteries. 
TABLE 2

The sites of origin of the thoraco-acromial artery

\begin{tabular}{|c|c|c|c|c|c|c|}
\hline Origin & Huelke & $\begin{array}{c}\text { DeGaris and } \\
\text { Swartley }\end{array}$ & $P^{\prime}$ an 1 & $\begin{array}{l}\text { Trotter } \\
\text { et al. } 1\end{array}$ & Adachi $^{1}$ & Pellegrini ${ }^{1}$ \\
\hline Axillary a. & $\%$ & $\%$ & $\begin{array}{c}\% \\
100.0\end{array}$ & $\begin{array}{c}\% \\
99.7\end{array}$ & $\begin{array}{l}\% \\
\text { extremely } \\
\text { constant }\end{array}$ & $\begin{array}{c}\% \\
82.5\end{array}$ \\
\hline $\begin{array}{l}\text { First part } \\
\text { Second part }\end{array}$ & $\begin{array}{l}29.8 \\
68.5\end{array}$ & $\begin{array}{l}85.7 \\
12.1\end{array}$ & & & & \\
\hline $\begin{array}{l}\text { Lateral Thoracic a. } \\
\text { Subscapular a. } \\
\text { Brachial a. }\end{array}$ & 0.6 & 1.2 & & & & 13.6 \\
\hline $\begin{array}{l}\text { Other } \\
\text { Absent }\end{array}$ & 1.1 & 1.0 & & & & 3.9 \\
\hline
\end{tabular}

${ }^{1}$ See footnote 2 , table 1 .

TABLE 3

The sites of origin of the lateral thoracic artery

\begin{tabular}{|c|c|c|c|c|c|c|}
\hline Origin & Huelke & $\begin{array}{l}\text { DeGaris and } \\
\text { Swartley }\end{array}$ & P'an ${ }^{1}$ & $\begin{array}{l}\text { Trotter } \\
\text { et al. } 2\end{array}$ & Adachi & Pellegrinin ${ }^{1}$ \\
\hline & $\%$ & $\%$ & $\%$ & $\%$ & $\%$ & $\%$ \\
\hline $\begin{array}{l}\text { First part of axillary a. } \\
\text { Directly }\end{array}$ & 10.7 & & & & & \\
\hline $\begin{array}{l}\text { Thor.-acrom a. } \\
\text { Sup. thor. a. }\end{array}$ & 2.8 & 36,5 & 0.7 & & & 1.0 \\
\hline $\begin{array}{l}\text { Second part of axillary a. } \\
\text { Directly }\end{array}$ & 52.2 & 55.9 & 71.4 & 69.6 & 40.0 & 70.2 \\
\hline $\begin{array}{l}\text { Thor.-acrom a. } \\
\text { Subscap. or }\end{array}$ & 3.9 & 6.4 & 1.4 & 7.0 & & 14.4 \\
\hline $\begin{array}{l}\text { thor. dorsal a. } \\
\text { Third part of axillary a. } \\
\text { Directly } \\
\text { Subscap, or }\end{array}$ & $\begin{array}{r}14.1 \\
1.7\end{array}$ & 1.2 & & & $60.0^{3}$ & 7.7 \\
\hline $\begin{array}{l}\text { thor, dorsal a. } \\
\text { Other } \\
\text { Absent }\end{array}$ & 14.6 & & 26.4 & 23.4 & & $\begin{array}{l}1.0 \\
5.8\end{array}$ \\
\hline
\end{tabular}

1 See footnote 2, table 1 .

2 Although not indicated in their report, it is assumed that the axillary and thoracoacromial origin of the lateral thoracic artery is behind the tendon of the pectoralis minor muscle and that the origin of the subscapular artery is from the third part of the axillary artery.

3 "It (the lateral thoracic artery) arises in $60 \%$ with other branches (usually with the subscapular a., sometimes with the thoraco-acromial a., seldom with the superior thoracic a.)".. Adachi.

the second part of the axillary artery, it is more often a direct branch than a branch of the thoraco-acromial or subscapular arteries. However, in approximately $43 \%$ of their cases, they found that the lateral thoracic artery (called thoracic branches in their study) arose from the thoracoacromial artery. In most of these cases the thoraco-acromial artery had an origin from the first part of the axillary artery. Only rarely did they find that the lateral thoracic artery arose from the subscapular artery. P'an and Trotter et al. found that the lateral thoracic artery arose directly from the second part of the axillary artery in approximately 7 out of 10 cases. In one-fourth of their sides, they found the lateral thoracic artery to be a branch of the subscapular or thoracodorsal artery. This figure-25\% - agrees closely with the data of my series (table 3). Pellegrini likewise found the lateral thoracic artery to have a direct origin in 7 out of 10 sides, all of which arose from the second part of the axillary artery. Furthermore, an origin from the thoraco-acromial trunk was found more often (14.4\%) than one from the subscapular or thoracodorsal arteries 
$(7.7 \%)$. In Pellegrini's study, no lateral thoracic arteries had an origin from the third part of the axillary artery or from its subscapular or thoracodorsal branches. Adachi found that the lateral thoracic artery was a direct axillary branch in only $40 \%$ of sides and that in $60 \%$ it arose from a common stem with the subscapular or other arteries.

The subscapular artery was usually the largest branch of the axillary artery, being the common stem for the circumflex scapular and thoracodorsal arteries in almost all cases. When the subscapular artery was absent $(1.7 \%)$, the circumflex scapular artery arose directly from the third part of the axillary artery and the thoracodorsal artery was a branch of the lateral thoracic artery or it had a separate and direct origin from the axillary artery. The subscapular artery had an origin from the third part of the axillary artery in $79.2 \%$ (fig. 1A). It arose from the second part of the axillary artery in $15.7 \%$ of sides (fig. 1B). Rarely it arose from the first part of the axillary $(0.6 \%)$ or from the deep brachial artery $(2.8 \%)$. The latter origin was quite unusual and only occurred when the second part of the axillary artery divided into the brachial and deep brachial arteries. The subscapular artery gave rise to the Iateral thoracic artery in one-fourth of all sides and to the posterior circumflex humeral artery in $15.2 \%$ (tables 3 and 4 ).
DeGaris and Swartley found the subscapular artery to be present in almost all cases. In their study the subscapular artery almost always $(94.1 \%)$ arose from the third part of the axillary artery and only rarely from the second part $(5.1 \%)$. P'an and Trotter et al. found the subscapular artery to be a direct axillary branch in all cases; however, the part of the axillary artery which was the more frequent site of origin was not given in either study. In approximately one-half $(46.4 \%)$ of his series, P'an found at least one named axillary branch arising from the subscapular artery (the anterior or posterior circumflex humeral arteries or the lateral thoracic artery). Pellegrini also found that the subscapular artery usually arose directly from the third part of the axillary artery $(95.2 \%)$. Adachi indicated that the subscapular artery was a direct branch of the axillary artery in $91.7 \%$ of cases. In the remainder of this series it was absent the circumflex scapular and thoracodorsal arteries arose separately.

The posterior circumflex humeral artery arose from the third part of the axillary artery in $78.7 \%$ of sides, usually as an independent branch (fig. 1A). It branched from the subscapular artery less frequently (15.2\% , fig. 1B) and rarely arose from any other source (table 4). The origin of the posterior circumflex humeral artery was usually proximal to that of the anterior circumflex humeral artery. However in

TABLE 4

The sites of origin of the posterior circumflex humeral artery

\begin{tabular}{|c|c|c|c|c|c|c|}
\hline Origin & Huelke & $\begin{array}{l}\text { DeGaris and } \\
\text { Swartley }\end{array}$ & P'an & $\begin{array}{l}\text { Trotter } \\
\text { et al. }\end{array}$ & Adachi & Pellegrini \\
\hline Third nart of axillary a & $\%$ & $\%$ & $\%$ & $\%$ & $\%$ & $\%$ \\
\hline $\begin{array}{l}\text { Third part of axillary a. } \\
\text { Directly }\end{array}$ & 67.5 & 73.4 & 35.7 & 63.3 & 33.0 & 37.5 \\
\hline $\begin{array}{l}\text { Common stem for } \\
\text { both humeral aa. }\end{array}$ & 11.2 & 15.8 & 27.9 & 22.9 & & 21.2 \\
\hline $\begin{array}{l}\text { Subscapular a. } \\
\text { Directly } \\
\text { Common stem for }\end{array}$ & 15.2 & 1.4 & 28.6 & 13.3 & 39.8 & 22.1 \\
\hline $\begin{array}{l}\text { both humeral aa. } \\
\text { Deep brachial a. }\end{array}$ & & & 5.0 & 0.5 & & 2.9 \\
\hline $\begin{array}{l}\text { Directly } \\
\text { Common stem for }\end{array}$ & 2.8 & 1.4 & 2.1 & & 27.2 & 11.5 \\
\hline both humeral aa. & 1.1 & 3.3 & 0.7 & & & \\
\hline Other & 2.2 & 4.7 & & & & 4.8 \\
\hline
\end{tabular}


$28.1 \%$ of sides it arose distal to the anterior circumflex humeral artery.

Most authors have reported that the posterior circumflex humeral artery usually was a branch of the third part of the axillary artery rather than from the subscapular artery (table 4). Adachi, however, stated that it arose in two-fifths of his sides from the subscapular artery and in only one-third of the sides from the terminal part of the axillary artery. Also contrary to the other investigations, Adachi's data showed the posterior circumflex humeral artery to be a branch of the deep brachial artery in $27.2 \%$. Pellegrini likewise found this type of origin to be more frequent $(11.5 \%)$ than most of the other authors.

In general, P'an, Adachi, and Pellegrini do not agree with the findings of the other studies on the origin of the posterior circumflex humeral artery. Possibly racial difference is the explanation, for P'an, and Adachi used oriental cadavers in their studies. Pellegrini, however, recorded his data from Italian cadavers, indicating that the origin of the posterior circumflex humeral artery is statistically different between nationalities, in this case Italians (Pellegrini) and American Whites and Negroes (material of this study, and that of DeGaris and Swartley, and Trotter et al.).

The anterior circumflex humeral artery was present on almost all sides (93.8\%) and rarely arose from any source other than the third part of the axillary artery (table 5). It was more frequently a direct branch of the axillary artery (80.3\%, fig. 1A) than from a trunk common to it and the posterior circumflex humeral artery $(11.2 \%)$. The findings of this investigation concerning the anterior circumflex humeral artery agree closely with previous reports (table 5). The occurrence of a common origin from the axillary artery for the anterior and posterior circumflex humeral arteries is variable. It was seen on $11.2 \%$ of sides in this study and ranged up to $27.9 \%$ in the series of P'an.

The unnamed branches of the axillary artery are small; however, one of these branches was quite constant $(86 \%)$. This artery can only be identified with assurance in its terminal course for its origin is variable. It supplies part of the subscapularis muscle, entering it through a neurovascular hilum with the upper subscapular nerve. Other arterial branches also supply the subscapularis muscle, but this is the only artery which enters the muscle in company with the upper subscapular nerve. Because of its high frequency it warrants recognition as a named branch. Since this artery is closely related to the upper subscapular nerve, it is suggested that it be named the "upper subscapular artery." In $51.6 \%$ of sides, the upper subscapular artery arose directly from the underside of

TABLE 5

The sites of origin of the anterior circumflex humeral artery

\begin{tabular}{|c|c|c|c|c|c|c|}
\hline Origin & Huelke & $\begin{array}{l}\text { DeGaris and } \\
\text { Swartley }\end{array}$ & P'an & $\begin{array}{l}\text { Trotter } \\
\text { et al. } 1\end{array}$ & Adachi & Pellegrini \\
\hline Third part of axillary a. & $\%$ & $\%$ & $\%$ & $\%$ & $\%$ & $\%$ \\
\hline & 80.3 & 75.0 & 62.9 & 76.6 & 66.8 & 69.2 \\
\hline both humeral aa. & 11.2 & 15.8 & 27.9 & 22.9 & 17.4 & 21,2 \\
\hline $\begin{array}{l}\text { Subscapular a. } \\
\text { Directly } \\
\text { Common stem for } \\
\text { both humeral aa. }\end{array}$ & & 1.2 & 5.0 & 0.5 & 4.5 & 2.9 \\
\hline $\begin{array}{l}\text { Deep brachial a. } \\
\text { Directly } \\
\text { Common stem for } \\
\text { both humeral aa. }\end{array}$ & 1.7 & 3.3 & 1.4 & & 4.5 & 1.9 \\
\hline $\begin{array}{l}\text { Other } \\
\text { Absent }\end{array}$ & $\begin{array}{l}0.6 \\
6.2\end{array}$ & 4.7 & 0.7 & & 5.8 & 4.8 \\
\hline
\end{tabular}

1 See footnote 2 , table 1 .

2 See footnote 1 , table 4 . 
the axillary artery : $30.3 \%$ from the second part of the axillary artery (fig. 1A), $17.4 \%$ from its first part, and $3.9 \%$ from its third part. The artery passed either over or under the posterior cord of the brachial plexus to reach the subscapularis muscle. It arose from the subscapular artery in $21.4 \%$ (fig. 1B), and from the lateral thoracic artery or from the third part of the subclavian artery in $4.5 \%$ each. In a total of $4 \%$ of sides it arose from the transverse cervical, suprascapular, or dorsal scapular arteries. The upper subscapular artery was absent on $14 \%$ of sides.

Among other authors only Pellegrini described an artery, having a variable origin, which supplies the subscapularis muscle (other than the usual branches from the subscapular artery). He indicated that this artery is present in $85 \%$ of cases.

Other unnamed branches of the second or third parts of the axillary artery passed to the subscapularis muscle in addition to the upper subscapular artery. These vessels occurred in $12.4 \%$ of sides. In addition, small, single or multiple twig-like arteries passed to the tendon common to the coracobrachialis and short head of the biceps brachii muscles near its coracoid attachment. They were seen in a total of $53.3 \%$ of sides and arose most frequently from the proximal end of the third part $(45 \%)$, or from the second part of the axillary artery ( $8.3 \%)$.

\section{The number of axillary branches}

From the foregoing analysis of the data it will be noted that 7 arteries may be considered as typical axillary branches. These branches are: the superior thoracic, thoraco-acromial, lateral thoracic, subscapular, anterior and posterior circumflex humeral, and the upper subscapular artery. Each branch, however, does not always arise directly from the axillary artery, and thus the total number of the direct axillary branches varies. Six factors may change the number of axillary branches. They are: (1) the complete absence of one or more of the branches; (2) branches arising from another axillary branch; (3) branches arising by a common stem; (4) branches arising from a source other than the axillary artery or one of its branches; (5) shortening of the axillary artery by dividing, at the lateral border of the pectoralis minor tendon into brachial and deep brachial arteries; (6) a combination of two or more of the above factors.

The number of axillary branches was studied on 150 sides. All 7 branches arose directly from the axillary artery in onefourth of the sides studied $(26.7 \%)$. Six axillary branches occurred most often $(37.3 \%)$. Five axillary branches occurred in 24.7 of sides. Four, three, and two axillary branches occurred in a total of $11.4 \%$ - (4 branches, $8.7 \%$; three branches, $2 \%$; two branches, $0.7 \%$ ).

The combination of the branches is also variable. Axillary arteries with 7 branches include all of the typical axillary branches. Axillary arteries with 6 branches arising directly exhibited 7 different combinations of the branches. In $17.4 \%$ all of the branches arose directly from the axillary artery except the upper subscapular artery which was either absent or had another origin. In $12 \%$ all branches except the lateral thoracic artery arose directly from the axillary artery. Either of these two groups occurred more frequently than any other grouping of the axillary branches. The other 5 groupings accounted for $8.1 \%$ of the sides. In these, all of the typical axillary branches arose from the main stem except the posterior circumflex humeral artery $(4 \%)$, anterior circumflex humeral artery $(0.7 \%)$, or the thoracoacromial artery $(0.7 \%)$. In $2 \%$ the circumflex humeral arteries arose by a common stem and in $0.7 \%$ the lateral thoracic artery arose from the thoraco-acromial artery with the subscapular artery being replaced by the scapular circumflex artery.

Axillary arteries with 5 branches had 9 different groupings of the branches. Four of these groupings accounted for most of the variables. In $4.7 \%$ the lateral thoracic artery arose from the thoracodorsal artery and the upper subscapular artery was absent; the posterior circumflex humeral and lateral thoracic arteries arose from the subscapular or thoracodorsal arteries in $4.7 \%$. In $4 \%$ neither the superior thoracic nor the lateral thoracic arteries arose directly from the axillary artery. In all of these three groupings the other branches arose directly from the axillary artery. In 
$4 \%$ the circumflex humeral arteries arose by a common stem, and the upper subscapular artery was absent or arose from some other artery. A similar pattern to the preceding one occurred in $2.7 \%$ of sides. Here the reduction in the number of branches was due to a common stem for the circumflex humeral arteries with the lateral thoracic artery arising from the subscapular artery. In one case $(0.7 \%)$ the scapular circumflex and thoracodorsal arteries arose separately from the axillary artery and the circumflex humeral arteries arose from the brachial artery-all other vessels were direct axillary branches. In the other three groups all arteries were direct axillary branches except the upper subscapular artery and the posterior circumflex humeral artery $(2 \%)$, the superior thoracic artery $(1.3 \%)$, or the anterior circumflex humeral artery $(0.7 \%)$.

Eight combinations of the branches were found in the axillary arteries with 4 branches. In two of these groups the reduction was due to the absence of a nonaxillary origin of the lateral thoracic, upper subscapular, and either the anterior circumflex humeral $(2 \%)$ or posterior circumflex humeral $(2.7 \%)$ arteries. In three other groups the circumflex humeral arteries arose from a common stem and either the lateral thoracic artery $(0.7 \%)$, the upper subscapular artery $(0.7 \%)$, or both of these arteries ( $0.7 \%)$ arose from the subscapular artery or were absent. In two groups the circumflex humeral arteries arose from the brachial artery and either the subscapular or the lateral thoracic artery was absent $(0.7 \%$ each). In one case $(0.7 \%)$ the superior thoracic, posterior circumflex humeral and upper subscapular arteries were absent.

All axillary arteries which had less than 4 branches were shorter than usual; in all of these cases the third part of the axillary artery was absent, for the artery divided into brachial and deep brachial arteries at the lateral border of the pectoralis minor muscle. Three axillary arteries had only three branches. In two of these the branches were the superior thoracic, the thoraco-acromial and the subscapular arteries. In both cases the anterior circumflex humeral artery was absent. In one case the lateral thoracic, pos- terior circumflex humeral, and upper subscapular arteries arose from the subscapular artery. In the other case the lateral thoracic and upper subscapular arteries arose from the subscapular artery and the posterior circumflex humeral artery arose from the brachial artery. In another case the three branches were the superior thoracic, thoraco-acromial, and lateral thoracic arteries. Here the subscapular and circumflex humeral arteries arose from the deep brachial artery with the upper subscapular artery arising from the subscapular artery.

Only one case of a two branched axillary artery was seen. In this case the axillary artery divided into the brachial and deep brachial arteries behind the pectoralis minor tendon. Only the superior thoracic and upper subscapular arteries arose from the axillary artery-all others arose from the deep brachial artery except the lateral thoracic and anterior circumflex humeral arteries which originated from the subscapular artery.

\section{CONCLUSION}

Seven named vessels may be considered as typical axillary branches, all of which were present in at least $86 \%$ of the sides examined. The superior thoracic artery arose from the first part of the axillary artery more often ( $87 \%$ ) than from any other source. The thoraco-acromial artery arises from the axillary artery near the medial border of the pectoralis minor muscle; an origin from the second part of the axillary artery was the more frequently seen (68\%). The lateral thoracic artery was found on $65 \%$ of sides as a distinct axillary branch, usually arising from the second part of the axillary artery ( $52 \%)$. It arose from the subscapular or thoracodorsal artery in $29 \%$. Both the subscapular and anterior circumflex humeral arteries arose from the third part of the axillary artery in 8 out of 10 sides. The posterior circumflex humeral artery was a direct branch of the third part of the axillary artery in $68 \%$ of sides, arising in $28 \%$ distal to the anterior circumflex humeral artery. In $11 \%$ of sides the circumflex humeral arteries arose by a common stem from the terminal part of the axillary artery. In $15 \%$ of sides the posterior circumflex humeral artery arose 
from the subscapular artery. A previously unnamed artery to the subscapularis muscle was present in $86 \%$ of cases. This vessel enters the muscle with the upper subscapular nerve. This "upper subscapular artery" arose directly from the axillary artery in one-half of all sides (52\%) and from the subscapular artery in $21 \%$. Other origins from the lateral thoracic or subclavian arteries, or from one of the scapular branches of the subclavian artery totalled $13 \%$. Other unnamed branches of the axillary artery are small twigs that supply both the coracobrachialis and the tendon of the short head of the biceps brachii muscle near their coracoid attachment, and the subscapularis muscle.

Although the axillary artery may have as many as 7 direct named branches, the 7 branches do not always have a direct axillary origin, for an origin of one or more of these branches from another source, or the absence of these vessels, reduces the number of the axillary branches. Six direct axillary branches were more frequently seen $(37 \%)$ than axillary arteries with $7(27 \%)$ or $5(25 \%)$ branches. In $11 \%$ of cases less than 5 direct axillary branches were seen. The combination of the branches is also variable. Axillary arteries with 4,5 , or 6 branches, where many combinations of the branches occur, had an average of 8 different arrangements of the branches.

\section{LITERATURE CITED}

Adachi, B. 1928 Das Arteriensystem der Japaner. Acta schol. med. Univ, imper. Kioto, 9: suppl. 1, 291-293, 307-316.

DeGaris, C. F, and W. B. Swartley 1928 The axillary artery in White and Negro stocks. Am. J. Anat., 41: 353-397.

Huelke, D. F. 1958 A study of the transverse cervical and dorsal scapular arteries. Anat. Rec., 132: 233-246.

$P^{\prime}$ an Ming-Tzu 1940 The origin of branches of the axillary artery in the Chinese. Am. J. Phys. Anthrop., 27: 269-279.

Pellegrini, A. 1906 Le arteriae subclavia e axillaris nell' uomo studiate col metodo statistico. Arch. Ital. Anat. Embriol., 5: 205 and 466.

Trotter, M., J. L. Henderson et al. 1930 The origins of branches of the axillary artery in Whites and American Negroes. Anat. Rec., 46: 133-137. 